\title{
1 Definitions and Description of Basic Terms
}

Sports preparation itself represents a complex system of phenomena, connections and behaviour of its components. If we want to reach the goal of sports preparation to nurture a top athlete able to reach an optimum performance in any conditions - we have to try, based on thorough knowledge of the individual and knowledge of specific age, sexual and developmental peculiarities, to create a model of rational long-term sports preparation which presupposes application of adequate and effective training means, methods and forms of work, optimum training loads and suitable frequency and follow-up in training cycles. To solve this crucial task for the trainer, the method of programming-modelling is used. To start to prepare a new model, it is practically necessary to define basic terms used in the book.

Modelling is a creative process resulting in the elaboration of optimum models. Through this, it is possible to describe phenomena and processes from the point of view of structure, functions, intentions of the course, as well as from the point of view of planning a possible and required development and final effect. The method is considered as one of the basic ways of investigating complex pedagogical phenomena as systems of intentional behaviour. The method of modelling is based on elaborating a model encompassing characteristic features of the personality of an athlete with levels of factors which limit sports performance in the given sport.

Modelling in sport is a creative process focused on designing optimum rational models of the long-term sports preparation, which also consider changes in athletes, developmental issues and external factors. The effect of model realization is on a higher level than the real model created by the input information. In sports, modelling is generally used in connection with:

- games modelling,

- modelling of conditions of games,

- working out models of long-term preparation, training units, micro-, mezo-, macro-cycles,

- modelling of characteristics of an ideal player,

- application of technical means and using of information (programmes of development of motor abilities, acquisition of rational sports technique and tactics).

A model is the result of the process of modelling and represents an idealized and materialized system which adequately reflects the object of investigation or analogously depicts its specific features and relations. In a broader sense, it represents an object deputizing other objects, which is able to offer information on them. A model presumes a precise notion of what factors and in which sequence such factors should be formed. It requires knowledge on how to create all the required features, abilities and skills determining the attainment of a top sporting performance. Moreover, it requires, an optimum contents, focus and progression of how to reach the final state in connection with the changes in individual factors 
of sports performance structure as to the age dynamism of their development. The development of individual factors should be planned in a rational way - through an optimum use of "sensitive periods", when the organism reacts to a specific training stimulus in the most optimum way and it is thus possible to reach the clearest changes during the shortest time (Šimonek, 1979).

Šimonek defines the model of an athlete as the picture of an ideal performer, a model depiction of ideal prerequisites of an athlete for reaching a certain performance goal. As a rule it includes information on somatic, motor, coordination-technical and personality prerequisites for reaching the set performance goal. From this point of view we can speak about a model of the talented performer - beginner, a model of the future Olympic winner, or a world record holder, but also an athlete of a certain performance level. Models of this kind have a great activating and motivating aim because they represent the target desired through sport preparation in volleyball.

Supposing that the models are as a rule oriented at final, target performances, from the point of view of the long-term sports preparation of young athletes, the so-called basal models are of greatest importance because they represent a sum of model characteristics of various aspects of preparedness of young sportspersons. At the end of the stage of expanded sports specialization in the selected sport, a young athlete should master a wide spectrum of motor habits and skills, reach a harmonious development of motor abilities - conditioning as well as coordination skills inevitable for a successful specialization and transition into adulthood.

Modern science in sports training emphasises models in which the top level focuses on general and special physical preparation, technical and tactical mastery, while reaching the planned sports performance should be based on a fundamental basic functional and coordination preparation.

Table 1.1 shows the position of indicators of functional preparedness of a sportsperson according to the importance for the particular kind of sport. In sport games we can find analysers in the first level of importance, while in the second one these are functional systems of the organism. It is inevitable to focus our attention to these factors in the sports preparation of children and youth.

In sports preparation, modelling is mostly used for the solution of the following tasks:

1. Prediction of the result of the particular training process, i.e. how the situation will be changed after the performance of the planned training.

2. Method of proceeding in the given situation so that we modify it in the desirable direction, i.e. to reach a change from starting situation to the terminal one.

In the sphere of top sport, coaches use models of the long-term sport preparation. In these models they aim to bring an athlete from the initial position into the terminal (desired) one. Models of long-term sport preparation are dynamic developing systems which apart from being informatory, also inspire meaning and provide a way to elaborate plans for the sports preparation of particular athletes, in particular kinds of 
Table 1.1: Distribution of indicators of functional preparedness of an athlete as to their importance (Nabatnikovová, 1982)

\begin{tabular}{|c|c|c|c|c|c|}
\hline \multirow{2}{*}{$\begin{array}{l}\text { Level } \\
\text { of } \\
\text { importance }\end{array}$} & \multicolumn{5}{|c|}{ Groups of sports } \\
\hline & Explosive & Cyclic & $\begin{array}{l}\text { Demanding } \\
\text { a high } \\
\text { degree of } \\
\text { coordination }\end{array}$ & Martial arts & Sports games \\
\hline I & 1,2 & $1,5,7,8,9$ & $1,2,3,6$ & $1,2,3,6$ & $1,2,3,4$ \\
\hline II & 6 & $2,3,6,10$ & $4,5,7,8,9$ & $5,7,8,9$ & $5,6,7,8,9$ \\
\hline III & 5 & 4 & 10 & 4,10 & 10 \\
\hline IV & 3,4 & & & & \\
\hline
\end{tabular}

Explanations: 1 - kinesthetic analyser, 2 - vestibular analyser, 3 - visual analyser, 4 - acoustic analyser, 5 - endocrine system, 6 - peripheral muscle-nerves system, 7 - cardio-vascular system, 8 - respiratory system, 9 - system of metabolism, 10 - thermal regulation system.

sports. Specific plans must respect age, sexual, and individual peculiarities, as well as social and material condition. The quality of the model always depends on the scope and level of knowledge used in the creation of the model.

In order to create a model of long-term sport preparation in volleyball, it is essential to devise the necessary fundaments, represented as follows:

1. The structure of sports performance in volleyball.

2. Age dynamism of the development of factors of the structure of sport performance, as well as intergender and individual peculiarities of the development of individual factors of the sports performance.

3. Dynamism of increasing training and competitive loads, as well as increasing sports performance.

Sport training is a process of complex biological, psychological, and social adaptation, in which an athlete is systematically loaded by a set of specific stimuli, in order to improve reactions, sport form, develop motor abilities, personal qualities, acquiring knowledge, motor skills, tactical acting, behaviour and to improve sport mastery.

Coordination can be defined as "cooperation of central nervous system and skeletal muscles within some aimed movement process” (Holmann \& Hettinger, 1990). Quality of coordination depends principally on processes of movement control and the connected nervo-muscular processes, as well as on the level of analysers. 
Movement coordination is defined as "temporal, spatial and power control of individual movements or complex motor expressions, which are executed with regard to tasks and goals handed over through senses" (Mechling, 1983).

Coordination abilities. Hirtz (1985) defines coordination abilities as "complex, relatively independent prerequisites of performance regulation of movements, which are created and developed in motor activities based on dominant, inherited but influenceable neuro-physiological functional mechanisms and therefore, they can be improved by means of a methodical training." Kirchem (1992) states that the terms "skill" and "agility" used before, are not able to explain the complexity of coordination abilities and to describe their structure. We deal with the problem of relationship between coordination abilities and motor skills (technique) in other chapter.

Reaction speed is an ability to react quickly by an adequate (standard or nonstandard) movement activity on a certain stimulus (acoustic, optic, tactile, kinesthetic) or actual change of situation (Hirtz, 1985). Impulse can be also a moving object (ball, puck, team-mate, opponent). We differentiate between a simple and complex motor reaction. In sport games complex motor reaction (reaction with an option) is the most common requirement; this requires fast selection from various options of such motor reaction, depending on which is most adequate and effective for the given situation, and which would lead to success with the largest probability. Gamble (2013) states that perceptionaction coupling and decision-making are critical elements in terms of developing the ability to express reaction speed and agility capabilities under match conditions.

Kinesthetic-differentiation ability is an ability to control movements in time, space and dynamics, which allows for reaching a high precision and fine harmonizing of individual phases, as well as movement activities as a whole. It requires conscious and perfect harmonization of the movement with the motion conception. Among the specific expressions of this ability belong "sense of movement", "sense of a ball or a puck", "sense of tempo" (Hirtz, 1985).

Space-orientation ability is an ability to learn fast and adequately change the position and movements of the body in space in relation to the external environment (court lines, team-mates, opponent, ball, goal) (Hirtz, 1985). This enables the player to have accurate orientation in any game situation and coordinate movements in compliance with the real movement task. It depends to a great degree on the quality of vestibular apparatus.

Rhythmic ability is an ability to grasp and simulate temporal and dynamic segmentation of the course of movement (Hirtz, 1985). We speak mostly about accomodation of the movement to the given (external) rhythm or finding an optimum and effective internal rhythm, allowing for reaching higher effectiveness of motor activity. Related to this also is the ability to accomodate to the motor rhythm of other athletes, team, to change rhythm of playing, to enforce one's own rhythm to the opponent.

Balance ability is an ability of an individual to maintain or restore balance of the body in situations where a fast or unexpected change in body position occur (Hirtz, 1985). Balance involves a host of sensorimotor capacities, comprising input from 
visual, vestibular and somatosensory systems (Bressel, Yonker, Kras, \& Heath , 2007). It plays an important role especially in ice-hockey. It depends on the size of the weightbearing surface, position of the body's centre of gravity, state of vestibular system and the CNS. Information from the vestibular systems is extremely important in terms of maintaining balance. We differentiate static and dynamic balance (from the point of view of sport games a high level of dynamic balance is required).

Besides the above mentioned 5 elementary coordination abilities there exist also other coordination abilities defined by various German authors:

Ability to redesign the motor programme. This ability allows for adjusting or rebuilding the original programme of operations during the performance (shooting at a goal, dribbling) based on perceived or expected changes to a situation, or to replace the motor programme by an adequate activity and to execute it (Schnabel \& Thiess, 1993). Corrections can be carried out based on the change of situation and can be expected or unexpected, induced by the action of the opponent or team-mate (fighting for a rebound puck, getting loose from the opponent for a teammate). Changes of situation lead most frequently to the change of one parameter of movement. Ability to rebuild occurs, for example, in all operations and following interception actions between a defence and an offence players.

Ability to couple phases of movement. This ability ensures spatial, temporal and dynamic accuracy of movements of parts of the body within the movement of the whole body aimed at certain movement target while contacting a ball and the opponent (Schnabel \& Thiess, 1993). It is manifested in an effective bonding of simple movement phases, or game activities into a complex sport activity, in partial movements executed either consecutively or simultaneously, uninterrupted but targeted, fluently and interconnectedly.

Agility. This term comprises the ability to stop, rapidly change direction, and accelerate in response to an external cue. Agility is required by many sports (Bloomfield, Polman, O`Donoghue \& McNaughton, 2007; Gabbett, Kelly \& Sheppard, 2008; Little \& Williams, 2005). Some literature uses the term quickness synonymously with agility or change-of-direction speed (Moreno, 1995; Sheppard \& Young, 2006). However, Sheppard and Young (2006) suggested that the definition of quickness does not consider deceleration or a change of direction and that quickness in and of itself contributes to agility. The literature indicates that agility must consider not only speed but also the ability to decelerate, change direction, and reaccelerate in response to stimuli. Agility is thus, a complex set of independent skills that converge for the athlete to respond to an external stimulus with a rapid deceleration, change of direction, and reacceleration (Sheppard \& Young, 2006; Young, James, \& Montgomery, 2002). These experts suggest that agility is affected by the athlete's perceptual and decision-making ability and the ability to quickly change the direction.

Eye-hand coordination (also known as hand-eye coordination) is the coordinated control of eye movement with hand movement, and the processing of visual input to guide reaching and grasping along with the use of proprioception of the hands 
to guide the eyes. If you want to learn how to improve eye-hand coordination, you are tuning a fine motor skill that develops shortly after birth. It is an important task regardless of your age. Children are taught how to improve eye-hand coordination as early as 4 months old through exploring of toys and games. Practicing this skill not only helps with daily activities or with a specific athletic sport you are trying to improve upon but it can also help delay the aging process.

Vision is the process of understanding what is seen by the eyes. It involves more than simple visual acuity (ability to distinguish fine details). Vision also involves fixation and eye movement abilities, accommodation (focusing), convergence (eye aiming), binocularity (eye teaming), and the control of hand-eye coordination. Most hand movements require visual input to be carried out effectively. For example, when children are learning to draw, they follow the position of the hand holding the pencil visually as they make lines on the paper. Between four and 14 months of age, infants explore their world and develop hand-eye coordination, in conjunction with fine motor skills. Fine motor skills are involved in the control of small muscle movements, such as when an infant starts to use fingers with a purpose and in coordination with the eyes.

\subsection{Modelling the Sport Preparation in Volleyball}

Elaboration of the model of sport preparation is a difficult, complex task requiring thorough knowledge of the reality in this kind of sport, as well as honest preparation for its realization. Since motor activity in volleyball is of a non-standard character, it is very difficult to create a serious model of sport preparation. Long-term sport preparation through application of an optimum focus and content of preparation, should ensure a gradual development of all those factors of the structure of sport performance, which condition sport performance to a crucial degree.

From this point of view, it is inevitable to know all the factors which form the structure of sport performance in volleyball. Modelling the preparation in sport games requires applying optimum focus and content of preparation, procedure of reaching this target status, as well as information on desired changes of individual factors of performance in compliance with age-related developmental changes.

An important prerequisite of effectiveness of the model of coordination preparation is the prognosis and application of training loads, optimum in volume, intensity, coordination complexity and psychological demands, as well as a gradual and sufficiently progressive increase in individual stages of the long-term sport preparation.

To summarize, it is possible to say that among the crucial prerequisites for the elaboration of the model of coordination preparation in volleyball belong:

- The knowledge of limiting factors of the structure of sport performance;

- The knowledge of the growth of sport performance and the decisive factors of its structure in the course of the long-term sport preparation; 
- The knowledge of dynamism of increase of load in the course of the long-term sport preparation.

\subsection{Characteristics of the Sport Preparation in Sport Games}

A constituent part of sport training in sport games is the fulfilment of various tasks which are called components of sport preparation. Only acquisition of all the components, which create the whole complex mosaic of the process that is called sport training, can lead to an optimum growth of performance level.

Individual components are represented in sport training in various ratios depending on the period in which the athlete is situated. When the sportsperson is in full preparation for competition or a tournament, the preparation contains predominantly technical and tactic means and in the period one or two months prior to the beginning of the competition period there prevails conditioning. However, it is important not to forget about maintaining a certain level of conditioning, as well as coordination abilities, even during the competitive period. This factor is frequently underestimated by coaches and also players, who refer mostly to fatigue and fear of overtraining. An important role is also played by the quality of recuperation processes.

At present, the classical division into preparatory, competitive and transition periods in certain sports is rather old-fashioned. It is due to the fact that in sport games like tennis (outdoor and indoor, winter and summer tournaments) or volleyball (competitions, tournaments, cups and national and international competitions, beach volleyball in summer) the competitive period lasts all the year round. Of course, we speak mainly about top-class or elite sport.

In the preparation of youth sport we can remain at "classical division", therefore, we consider it important to know the inherent laws of training periods and training components.

The preparatory period consists of two stages. The task of the preparatory period in the first stage (preparatory period 1) is to create functional prerequisites for further growth of performance. The basic task of preparation is to increase condition by means of overall and special conditioning. Training is mostly focused on the development of aerobic capacity of the organism. In this period, the means of overall and special physical preparation blend together in a certain ratio, see Table 1.2 which shows an outline representation of components of sport training in individual training periods.

Besides other components of sport preparation such as technical, tactical, theoretical, psychological preparation and medical observation, conditioning plays the most important role since it is the decisive determinant for all sport activities. The importance of conditioning is manifested in various age categories in different proportions. A core of conditioning is formed by the development of motor prerequisites 
Table 1.2: Components and periods of sport training in the year cycle in \% (Felix, 1997)

\begin{tabular}{lllll}
\hline Components of sport training & $\begin{array}{l}\text { Preparatory } \\
\text { period I. }\end{array}$ & $\begin{array}{l}\text { Preparatory } \\
\text { period II. }\end{array}$ & $\begin{array}{l}\text { Competitive } \\
\text { period }\end{array}$ & $\begin{array}{l}\text { Transition } \\
\text { period }\end{array}$ \\
\hline Overall conditioning & 70 & 10 & - & 90 \\
\hline Special conditioning & 20 & 50 & 10 & - \\
\hline $\begin{array}{l}\text { Technical and tactical } \\
\text { preparation }\end{array}$ & - & 35 & 75 & - \\
\hline $\begin{array}{l}\text { Psychological preparation } \\
\text { Theoretical preparation }\end{array}$ & - & - & 5 & - \\
\hline Medical observation & 5 & 5 & 5 & - \\
\hline
\end{tabular}

of an athlete. Reaching the level of sport performance is a long-term and multifactorial process, in which the development of motor abilities has an inseparable part. This growth is carried out based on adaptation changes being in progress in the particular physiological, functional systems and corresponding psychological processes and is conducted in cooperaton with the acquisition of motor skills and habits.

In compliance with Šimonek and Zrubák (1995) we can define conditioning as "pedagogical process focused on increasing functional and psychic possibilities of an athlete, strengthening health, overall physical growth and creation of such motor potential of an athlete, which shall alow him to reach a high level of sport mastery upon applying rational technique and tactics and upon optimum utilization of personal prerequisites".

From this definition it appears necessary to also specify separately“coordination preparation" although this did not exist in the past. Based on research, coordination abilities and conditioning abilities form at least equivalent prerequisites in humans for optimum motor activity and thus we assume that our reqirement to introduce a new term "coordination preparation" is eligible.

Conditioning plays the most important role in the training of children and youth where it fulfills two functions:

1. Overall physical preparation, which is focused on the development of functional possibilities of an organism based on general motor development,

2. Special physical preparation, focused on maximum development of motor abilities, limiting sport performance in the given sport specialization.

Overall and special conditioning preparation form a unit and that is why also overall preparation must be related to the given sport branch. Correctly focused conditioning can result in an optimum state of physical and psychic preparedness of an athlete according to Dovalil et al. (2002). The importance of conditioning is manifested 
in specific proportions in various ages, in various sport branches and on different performance levels.

Youth training requires a specific and quite different approach to design and implementation of physical preparation. As famously stated by Tudor Bompa, young people cannot merely be considered "mini adults" (Bompa, 2000). The physiological makeup of children and adolescents is markedly different from that of mature adults (Naughton et al., 2000) - it follows that the parameters applied to training design should reflect these differences.

The young athlete's neural, hormonal and cardiovascular systems develop with advances in biological age, leading to corresponding changes in neuromuscular and athletic performance (Quatman et al., 2006). Rates of development of a number of physiological and physical performance parameters measured in young team sports athletes are shown to peak at approximately the same time as they attain peak height velocity (Philippaerts et al., 2006). The age at which this occurs is highly individual; "typical" ages are around 11.5 years for females (Barber-Westin et al., 2006) and for males in the range of 13.8-14.2 years (Philippaerts et al., 2006). However, this can vary considerably - levels of biological and physiological maturation can be markedly different between young athletes of the same chronological age (Bompa, 2000; Kraemer \& Fleck, 2005).

In early childhood there prevails overall sport preparation (development of an overall fitness), with an increasing age and growth of sports performance the ratio between overall and special physical preparation equalizes and in the stage of top training special physical preparation represents only a small share (Tab. 1.3).

The specified shares of components of sport training which generally apply may be modified in different sports depending on the periodisation of sport preparation.

In volleyball, coaches must often solve the problem of improving the quality of physical preparedness of a player, when increasing the volume and intensity of loading does not bring the required effect. From this point of view, several experts have shown through experiments that the development of strength, speed, endurance and flexibility is enhanced when preceded by good quality goal-oriented coordination development. This, in turn, forms a base for reaching the level of sport mastery in the shortest time.

The level of coordination abilities in volleyball underlies markedly the quality, tempo and stability of the acquired sports skills, as well as their perfect utilization in game activities. Since the components of coordination develop mostly in the period before puberty, it means that the crux of the development of coordination abilities falls into the period specified by the stage of elementary sport preparation.

From the point of view of periodization of sport preparation, the specialized coordination preparation plays the most important role in the second preparatory period, when the athlete prepares for acquisition of technically demanding motor skills. 
Table 1.3: Ratio between generally developing and specialized exercises in the preparation of children and youth (Kampmiller, 1991)

\begin{tabular}{|c|c|c|c|c|c|c|c|c|c|c|c|}
\hline \multirow{2}{*}{$\begin{array}{l}\text { Components } \\
\text { of conditioning } \\
\text { preparation }\end{array}$} & \multicolumn{11}{|c|}{ Age } \\
\hline & 9 & 10 & 11 & 12 & 13 & 14 & 15 & 16 & 17 & 18 & 19 \\
\hline $\begin{array}{l}\text { General sport } \\
\text { preparation }\end{array}$ & 85 & 85 & 80 & 70 & 65 & 60 & 40 & 30 & 20 & 10 & 5 \\
\hline $\begin{array}{l}\text { Special sport } \\
\text { preparation }\end{array}$ & 0 & 0 & 3 & 7 & 5 & 5 & 15 & 20 & 25 & 30 & 35 \\
\hline
\end{tabular}

Explanatory notes: Data on overall and special preparation specify percentage, which belongs to conditioning besides other components of sport preparation of a young athlete.

The substance of the special physical preparation in sport games can be seen initially in the development of coordination abilities of a player, which then determine not only quality but also degree of utilization of conditional factors. Analysis of activity in sport games shows that many athletes have shortcomings in individual technical prerequisites. Particularly in the conditions of a game, the player is not often able to master the technique in an optimum way and to utilize the experience obtained during the training. One of the reasons for this can be an insufficiently developed coordination base of an athlete. These inadequacies can be removed if a goal-oriented and systematic programme of development of coordination abilities is conducted in parallel with the skills acquisition phase. Knowing the coordination prerequisites limiting the performance in sport games is essential in order to increase sport preparedness of a player. 